
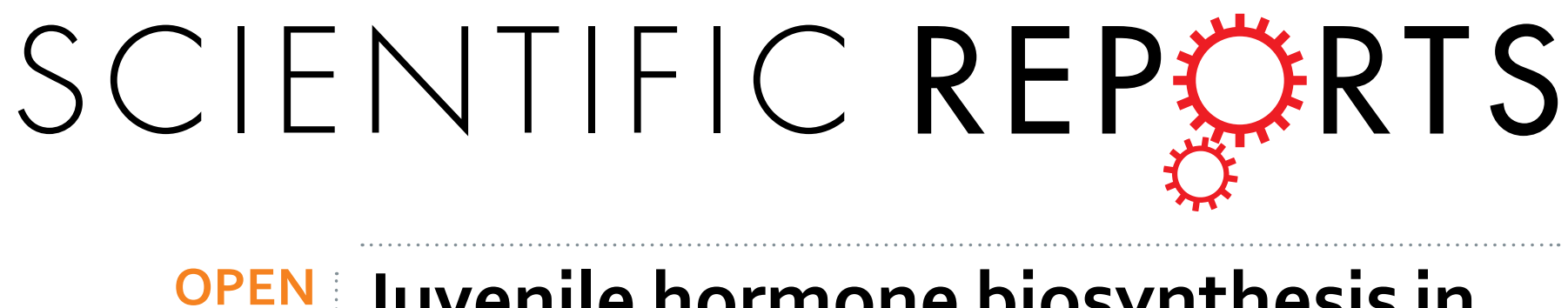

\title{
Juvenile hormone biosynthesis in adult Blattella germanica requires nuclear receptors Seven-up and
}

Received: 25 July 2016

Accepted: 02 December 2016

Published: 11 January 2017
FTZ-F1

\author{
Ferran Borras-Castells, Claudia Nieva, José L. Maestro, Oscar Maestro, Xavier Belles \& \\ David Martín
}

In insects, the transition from juvenile development to the adult stage is controlled by juvenile hormone (JH) synthesized from the corpora allata (CA) glands. Whereas a JH-free period during the last juvenile instar triggers metamorphosis and the end of the growth period, the reappearance of this hormone after the imaginal molt marks the onset of reproductive adulthood. Despite the importance of such transition, the regulatory mechanism that controls it remains mostly unknown. Here, using the hemimetabolous insect Blattella germanica, we show that nuclear hormone receptors Seven-up-B (BgSvp-B) and Fushi tarazu-factor 1 (BgFTZ-F1) have essential roles in the tissue- and stage-specific activation of adult CA JH-biosynthetic activity. Both factors are highly expressed in adult CA cells. Moreover, RNAi-knockdown of either BgSvp-B or BgFTZ-F1 results in adult animals with a complete block in two critical $\mathrm{JH}$-dependent reproductive processes, vitellogenesis and oogenesis. We show that this reproductive blockage is the result of a dramatic impairment of JH biosynthesis, due to the CAspecific reduction in the expression of two key JH biosynthetic enzymes, 3-hydroxy-3-methylglutaryl coenzyme A synthase-1 (BgHMG-S1) and HMG-reductase (BgHMG-R). Our findings provide insights into the regulatory mechanisms underlying the specific changes in the CA gland necessary for the proper transition to adulthood.

Postembryonic development in winged insects is divided into three stages: (i) a juvenile phase where growth takes place, (ii) the metamorphic transformation during the last juvenile instar, and (iii) the reproductive adulthood. Juvenile hormone $(\mathrm{JH})$, a sesquiterpenoid hormone produced and released by the corpora allata (CA) glands, controls critical events that occur during these three stages and also the transitions between them ${ }^{1-3}$. Thus, the presence of $\mathrm{JH}$ during pre-ultimate juvenile stages prevents the premature differentiation of adult features, while its disappearance upon entering into the last immature stage allows metamorphosis to occur ${ }^{4}$. Finally, the reappearance of high amounts of $\mathrm{JH}$ after the imaginal molt marks the onset of the adult period. During this stage, $\mathrm{JH}$ controls critical aspects of the reproductive process, such as yolk protein synthesis and oocyte maturation ${ }^{5-7}$. Despite the importance of the precise fluctuations of $\mathrm{JH}$ levels during insect development, the molecular mechanisms underlying the regulation of $\mathrm{JH}$ biosynthesis remain poorly understood.

$\mathrm{JH}$ biosynthesis involves a series of enzymatic reactions that are conventionally organized into two modules. In the first one, known as the mevalonate pathway, acetyl-CoA is transformed into farnesyl pyrophosphate, while the reactions in the second module are responsible for the conversion of farnesyl pyrophosphate into $\mathrm{JH}^{8}$. The genes encoding the enzymes involved in both modules are highly enriched, or even exclusively expressed, in the CA cells'. In the lepidopteran Bombyx mori, the dipteran Aedes aegypti and the dictyopteran Diploptera punctata, the expression patterns of JH biosynthesis enzymes in the CA cells correlate well to the JH synthetic activity of the gland, suggesting that the fluctuations in JH synthesis are mainly controlled at the level of the expression of these enzymes $^{9-13}$. Unfortunately, very few factors have been connected to the transcriptional control of JH synthesis to date. In the dipteran Drosophila melanogaster, TGFbeta signaling stimulates JH production by up-regulating the transcription of the JH acid methyltransferase (JHAMT) gene through the transcription factor Mothers against

Institute of Evolutionary Biology (CSIC-Universitat Pompeu Fabra) Passeig Marítim de la Barceloneta 37-49, 08003 Barcelona, Spain. Correspondence and requests for materials should be addressed to D.M. (email: david.martin@ ibe.upf-csic.es) 
Dpp (Mad) ${ }^{14}$. In the coleopteran Tribolium castaneum, the POU factor Ventral veins lacking/Drifter (vvl) activates $\mathrm{JH}$ synthesis during larval stages by activating JHAMT3 expression ${ }^{15}$. In addition to these transcription factors, several peptide hormones (allatotropins, allatostatins and short neuropeptide F) and neurotransmitters (the biogenic amines octopamine, dopamine and glutamate) are also involved in the regulation of CA activity ${ }^{16}$. However, although these factors regulate $\mathrm{JH}$ synthesis to some extent, the transcriptional regulatory network that control the expression of key JH biosynthetic genes during the different stages of development remains to be defined.

Here, we use the hemimetabolous insect Blattella germanica to elucidate the molecular mechanisms underlying the critical reactivation of $\mathrm{JH}$ production that marks the onset of the reproductive adulthood. This cockroach provides an ideal opportunity to address JH biosynthesis regulation as patterns and functions of JH III, the only $\mathrm{JH}$ form detected in this species ${ }^{17}$, have been intensively studied during the nymphal and adult stages ${ }^{18,19}$. The successive gonadotrophic cycles that conform the adult period of B. germanica females are characterized by a very high rate of JH synthesis, which induces the massive production of yolk precursor proteins by the fat body, as well as the incorporation of these proteins into developing oocytes ${ }^{7,20-23}$. Concomitantly with the strong increase in the rate of $\mathrm{JH}$ biosynthesis after the imaginal molt, the CA cells of $B$. germanica undergo a specific physiological change during this stage, switching from the proliferative phase characteristic of the juvenile development to an adult-specific non-proliferative state ${ }^{24,25}$.

In the present study, by using a detailed RNA interference (RNAi) analysis, we uncover the specific regulatory role of two transcription factors that controls JH synthesis and cell proliferation in the CA of adult B. germanica. Our findings reveal that nuclear hormone receptors Seven-up-B (BgSvp-B) and Fushi tarazu-factor 1 (BgFTZ-F1) provide tissue- and stage-specificity to the expression of two key JH biosynthetic enzyme genes, 3-hydroxy-3-methylglutaryl coenzyme A synthase-1 (BgHMG-S1) and 3-hydroxy-3-methylglutaryl coenzyme A reductase (BgHMG-R), in the CA cells to control the massive production of JH during B. germanica adulthood. We also provide evidence that BgSvp-B is required to maintain the proliferative capacity of CA cells during nymphal development. Thus, our work identifies the transcription factors that generate the physiological and functional changes in the CA gland necessary for the proper transition to the adult stage and the reproductive adulthood.

\section{Results}

Cloning and developmental expression of BgSvp isoforms. In search of transcription factors that might regulate the activity of CA cells in B. germanica, nuclear receptor Svp emerged as a good candidate as it has been found to be one of the few factors strongly and specifically expressed in the corpus allatum of D. melanogaster embryos $^{26,27}$. After a PCR step using degenerated primers designed in conserved regions of insect $S v p$ sequences and subsequent $5^{\prime}$ - and $3^{\prime}$-RACE-PCR methodologies, we obtained two cDNAs from B. germanica. The sequences, differing only in the C-terminal region, corresponding to the ligand binding domain (LBD), were named BgSvp-A (accession number: KT423097) and BgSvp-B (accession number: KT423098) based on the similarity of their DNA-binding domain (DBD) and LBD with other insect Svp (Supplementary Fig. S1). BgSvp-B has all the domains of the nuclear receptor family and shares more than $90 \%$ identity in the DBD and LBD with other insect Svp and with its vertebrate homolog, chicken ovalbumin upstream promoter transcription factor 1 (COUP-TF1). BgSvp-A is identical to BgSvp-B except for the last 90 amino acids of the LBD. Instead, BgSvp-A presents a short 21 amino acid stretch with no homology to other Svp proteins or known nuclear factors (Supplementary Fig. S1).

As a first step towards the characterization of $B g S v p$ functions, the mRNA levels of $B g S v p-A$ and $B g S v p-B$ were analyzed in different tissues of $B$. germanica. Consistent with a possible role in the CA, both BgSvp isoforms were strongly expressed in these glands. They were also detected in the fat body and brain although to a lesser extent, while no expression was detected in the other tissues analyzed (Fig. 1A). Given the tissue specificity, BgSvp isoforms expression was investigated further in the CA and found that $B g S v p-A$ and $B g S v p-B$ mRNAs persisted in these glands without major variations during B. germanica development (Fig. 1B).

BgSvp nuclear receptors play a central role in the adult stage of $\boldsymbol{B}$. germanica. To examine the role of BgSvp isoforms, we used systemic RNA interference (RNAi) to knockdown BgSvp during nymphal development. First, we lowered the expression of both receptors simultaneously by injecting a dsRNA encompassing the common LBD region ( $d s B g S v p-1$; Supplementary Fig. S2A). $d s B g S v p-1$ was injected in newly emerged last instar female nymphs (herein called $B g S v p i$ animals), and mRNA levels of $B g S v p-A$ and $B g S v p-B$ in the CA were determined 7 days later. Specimens injected with $d s M o c k$ were used as negative controls (Control animals). The mRNA levels of both receptors decreased substantially in BgSvpi insects compared to Control animals (75\% for $B g S v p-A$, and $81 \%$ for $B g S v p-B$ ) (Supplementary Fig. S2B,C).

All BgSvpi nymphs $(n=124)$ molted properly into adults and had normal appearance, as Control nymphs $(n=50)$ (Fig. 2A). This allowed us to analyze the role of BgSvp during the adult period. The first gonadotrophic cycle of $B$. germanica starts just after the imaginal molt and lasts 8 days, during which JH induces the synthesis of yolk protein precursors in the fat body. The yolk proteins are then released to the hemolymph and subsequently incorporated to the growing basal oocytes in a JH-dependent manner. As a result of the massive incorporation of yolk proteins, the basal oocytes show an exponential growth during each gonadotrophic cycle. Then, at the end of the cycle, eggs are oviposited into an egg-case or ootheca that is transported by the female during 18 days until egg hatching ${ }^{7,20-22}$. Notably, whereas all Control adult females oviposited eight days after the imaginal molt, none of the BgSvpi animals oviposited nor produced any sign of ootheca formation. A detailed examination of the BgSvpi females 5 days after the imaginal molt revealed a complete impairment of egg development (Fig. 2B,C). Given that oocyte growth depends on the accumulation of large amounts of the yolk protein vitellogenin (BgVg), we next measured the expression of $B g V g$ gene in the fat body of $B g S v p i$ adults. Compared to Control adults, $B g V g$ 
A

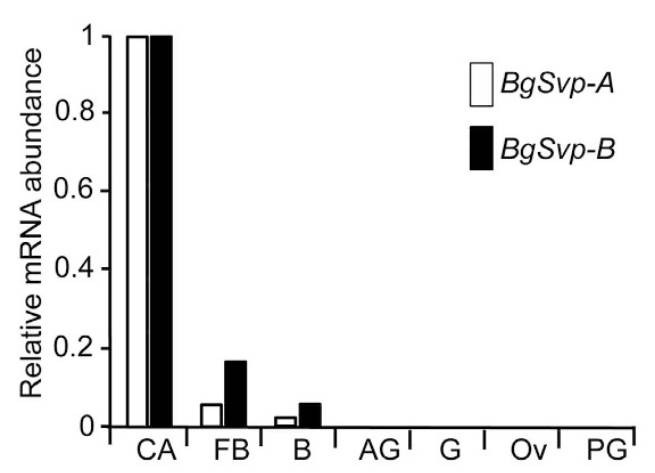

B

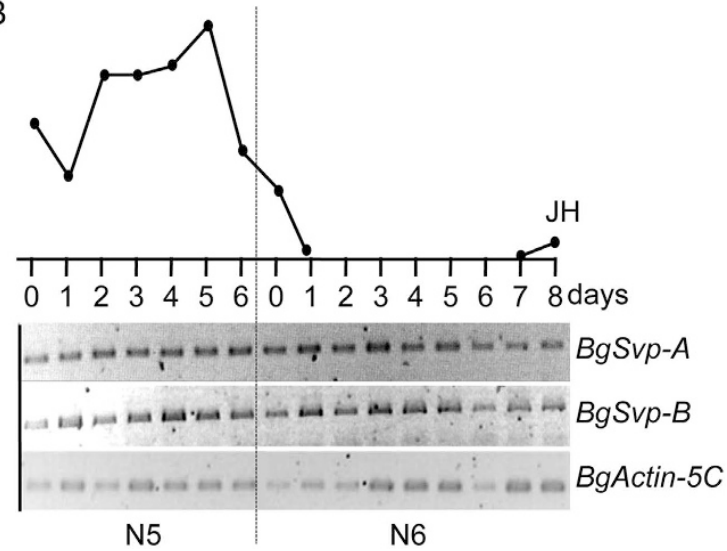

Figure 1. BgSvp isoforms are highly expressed in the CA of B. germanica. (A) Expression levels of BgSvp-A, and $B g S v p-B$ relative to $B g A c t i n 5 C$ in different tissues of 8-old-day B. germanica last instar nymph females, measured by qRT-PCR. corpora allata (CA), ovary (Ov), fat body (FB), brain (B), accesory glands (AG), prothoracic gland (PG), and gut $(G)$. Fold changes are relative to the expression of each gene in the CA, arbitrarily set to 1. (B) Expression patterns of $B g S v p-A$ and $B g S v p-B$ mRNAs in the CA during the last two nymphal instars of B. germanica, analyzed by semi-quantitative RT-PCR. BgActin $5 \mathrm{C}$ mRNA levels were used as a reference. The blot is representative of three replicates. Circulating JH levels (upper part) are redrawn from ref. 19.

mRNA levels in BgSvpi animals were dramatically reduced (Fig. 2D) and, consequently, BgVg protein was neither detected in the hemolymph nor within the oocytes (Fig. 2E). Altogether, our results show that BgSvp isoforms play a central role in the reproduction of $B$. germanica adult females.

In order to characterize whether the requirement of BgSvp was exclusive of the adult stage or it was also necessary during the pre-ultimate nymphal stages, $d s B g S v p-1$ was injected into the abdomen of freshly ecdysed fourth (antepenultimate) or fifth (penultimate) instar nymphs. Under these conditions, $100 \%$ of BgSvpi nymphs $(n=30)$ developed normally and underwent two or three successive molts, depending at which instar they had been injected, until reaching adulthood properly, indicating that $B g S v p$ isoforms are not involved in vital processes during nymphal development. Eventually, the BgSvpi adults showed the same reproductive impairment observed in BgSvpi animals injected in the last nymphal instar.

BgSvp-B is the isoform required for reproduction in adult $B$. germanica. To assess the role of each $B g S v p$ isoform on the $B g S v p i$ phenotype, dsRNAs targeting each isoform ( $d s B g S v p-A$ and $d s B g S v p-B$; Fig. S2A) were injected separately into newly emerged last instar nymphs ( $B g S v p-A i$ and $B g S v p-B i$ animals, respectively). The treatment resulted in a remarkably decrease in the corresponding transcript without affecting the expression of the other isoform (Fig. 3A). As in BgSvpi animals, all the isoform-specific knockdown nymphs developed normally and molted to the adult stage without any observable defect ( $n=30$ for $B g S v p-A i ; n=40$ for $B g S v p-B i$ ). After completing the first gonadotrophic cycle, Control and BgSvp-Ai females successfully oviposited while $B g S v p-B i$ females were unable to lay eggs as they showed a drastic impairment in egg development and reduced $B g V g$ expression in the fat body (Fig. 3B,C). Altogether, these results show that BgSvp-B is the responsible for the reproductive impairment observed in the BgSvpi animals.

BgSvp-B controls JH production by regulating the expression of $\mathrm{JH}$ biosynthetic enzyme genes. Given the high expression of $B g S v p-B$ in the CA and that the defects observed in $B g S v p-B i$ adults are suggestive of a JH deficiency, we next studied whether $B g S v p-B$ is crucial for controlling the biosynthesis and/ or the sensitivity to JH during the adult stage. To this aim, we first confirmed that $B g S v p-B$ was expressed in the CA cells of adult $B$. germanica females (Fig. 4A). Then, we measured JH production by the CA from 5 -day-old $B g S v p-B i$ adults and found that $\mathrm{JH}$ synthesis was drastically reduced compared to BgSvp-Ai and Control animals (Fig. 4B). To analyze whether JH sensitivity was also compromised in BgSvp-Bi adults, we topically applied methoprene, a potent $\mathrm{JH}$ mimic, in newly ecdysed $B g S v p-B i$ adults. This treatment restored normal levels of $B g V g$ expression in the fat body and the growth of the primary follicles (Fig. 4C,D), indicating that the adult phenotype of $B g S v p-B i$ animals is due to impaired JH synthesis but not to the transduction of the JH signal. Taken together, these results show that $B g S v p-B$ plays a critical role in the reproduction of B. germanica by controlling JH biosynthesis in the CA during the adult stage.

To address how BgSvp-B controls JH biosynthesis, we next asked whether it regulates the expression of genes encoding crucial enzymes of the pathway. In B. germanica, BgHMG-S1 and BgHMG-R have been identified as key enzymes in the mevalonate pathway leading to JH synthesis ${ }^{28-30}$. B. germanica has a second HMG synthase gene, $B g H M G-S 2$, although its expression and regulation is identical to $B g H M G-S 1^{30,31}$. Paralleling the rate of $\mathrm{JH}$ synthesis, mRNAs of $B g H M G-S 1$ and $B g H M G-R$ are significantly upregulated in the CA during the nymphal-to-adult transition and are maintained high during the first gonadotrophic cycle (Fig. 5A,B). Thus, we next examined whether $B g S v p-B$ depletion affects the expression of $B g H M G-S 1$ and $B g H M G-R$ during the adult stage. Remarkably, $B g H M G-S 1$ and $B g H M G-R$ mRNA levels in the CA of $B g S v p$ - $B i$ adults were severely reduced compared to BgSvp-Ai and Control adults (Fig. 5C). In contrast, mRNA levels of BgHMG-S1 and BgHMG-R in the 
A
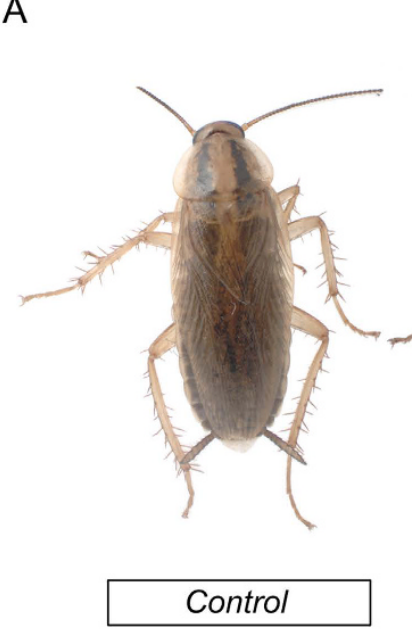

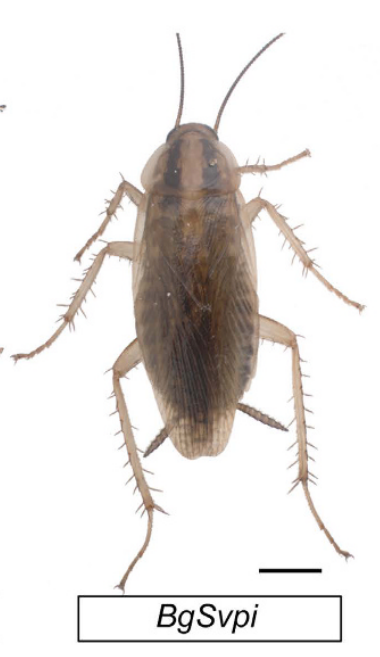

B
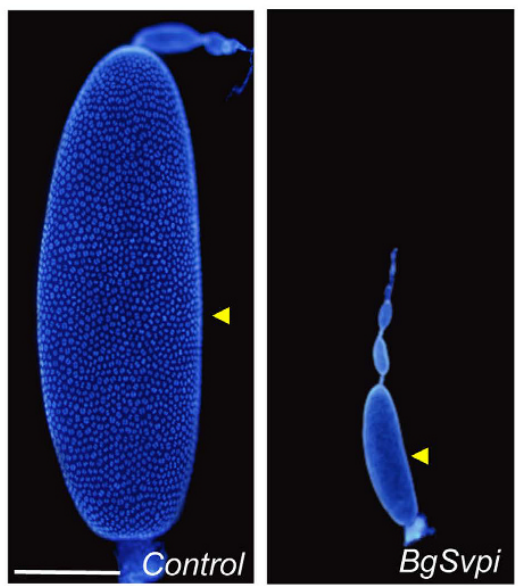

C

D
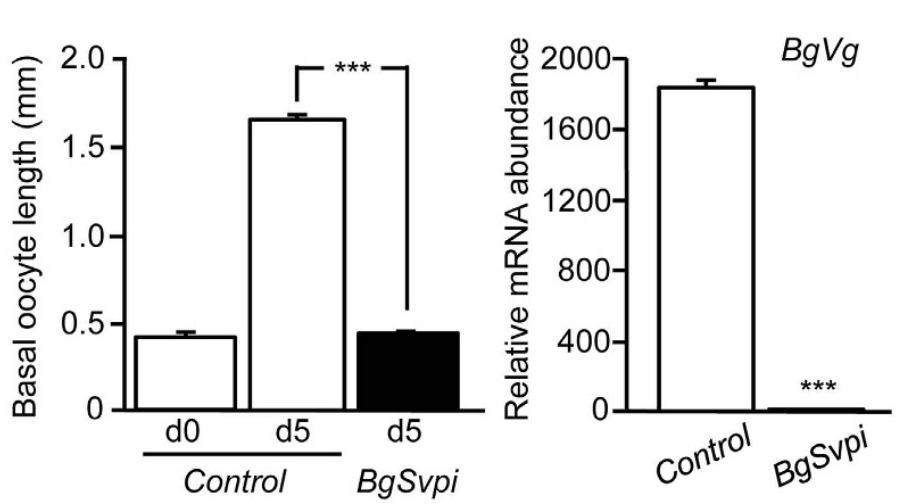

$\mathrm{E}$

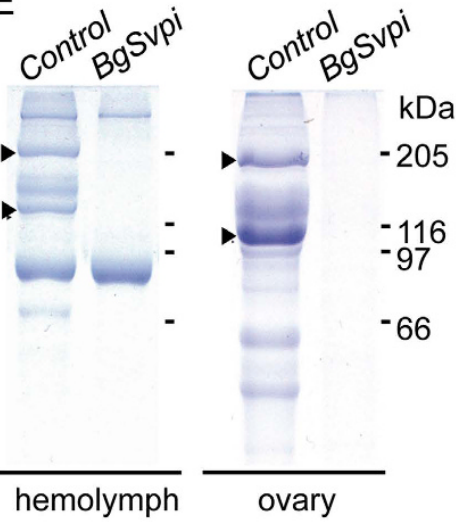

Figure 2. Loss of BgSvp impairs reproduction in B. germanica adult females. (A) Effect of RNAi of $B g S v p$. Newly emerged sixth instar female nymphs were injected with $3 \mu \mathrm{g}$ of $d s M o c k$ (Control) or $d s B g S v p-1$ (BgSvpi). Dorsal views of Control and BgSvpi animals showing normal winged adult appearance. (B) DAPIstained ovarioles from 5-day-old Control and BgSvpi adult females. Arrowheads indicate basal oocytes. (C) Basal oocyte length of Control and BgSvpi adult females of the indicated age. Results are expressed as the mean \pm S.E. $(\mathrm{n}=25-31)$. (D) $B g V g$ mRNA levels in the fat body of 5-day-old Control and BgSvpi females, relative to BgActin5C mRNA levels, measured by qRT-PCR. Error bars represent SEM $(\mathrm{n}=4)$. Asterisks in $(\mathrm{C}$ and D) indicate differences statistically significant as follows: ${ }^{* *} p \leq 0.0001$ (Student's $t$ test). (E) SDS-PAGE of hemolymph (left) and ovaries (right) from 5-day-old Control and BgSvp-1i adult females. Arrowheads indicate BgVg subunits. Scale bars: $1 \mathrm{~mm}$ in (A); $200 \mu \mathrm{m}$ in (B).

fat body of BgSvp-Bi adults did not differ from those in Control and BgSvp-Ai animals (Fig. 5D), suggesting that the regulatory effect of BgSvp-B is CA-specific. To confirm that the reduced levels of BgHMG-S1 and BgHMG-R in the CA were due to the absence of $B g S v p-B$ and not because of the low titer of $\mathrm{JH}$, we applied methoprene to newly ecdysed $B g S v p-B i$ adults and the mRNA levels of both enzymes were analyzed 2 and 5 days later. As Fig. 6 shows, methoprene was not able to induce BgHMG-S1 and BgHMG-R expression when BgSvp-B levels are reduced. Overall, our data show that BgSvp-B is necessary in adult CA cells for the specific up-regulation of, at least, two important JH biosynthesis enzyme genes, $B g H M G-S 1$ and $B g H M G-R$, in order to support the high rate of JH synthesis associated to adulthood in B. germanica.

BgSvp-B controls the proliferation of CA cells during nymphal development. The CA cells of $B$. germanica proliferate during the successive nymphal stages and switch to an adult-specific non-proliferative state immediately after the imaginal molt ${ }^{24,25}$ (Fig. 7A-D). Given the continuous and strong expression of $B g S v p-B$ in nymphal CA cells (Fig. 1B), we wondered whether BgSvp-B is involved in the control of CA cell proliferation during nymphal development. To test this possibility, we injected BrdU into staged last instar BgSvp-Bi and Control nymphs and analyzed its incorporation into CA cells $24 \mathrm{~h}$ later. Notably, CA cells from BgSvp-Bi nymphs did not show any BrdU-labeling in contrast to Control animals (Fig. 7E). The same result was obtained when BrdU incorporation was measured in CA cells of BgSvp-Bi penultimate instar nymphs (Fig. 7F). Overall, our data show that, in addition to its critical role in the transcriptional control of JH biosynthetic enzymes during the adult stage, BgSvp-B is also necessary for maintaining the continuous proliferation of the CA cells during nymphal development. 
A

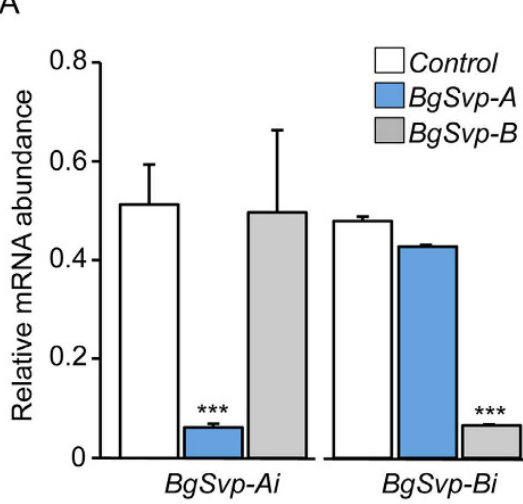

B

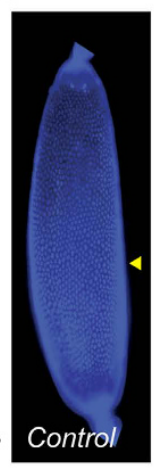

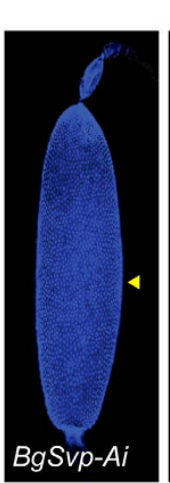

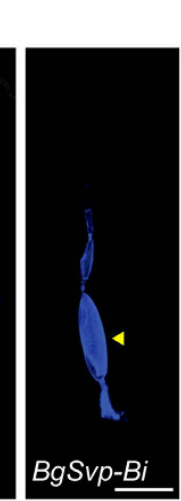

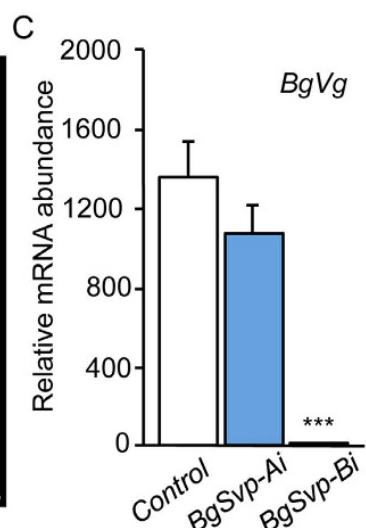

Figure 3. BgSvp-B is the isoform necessary for adult reproduction in B. germanica. (A-C) Effect of BgSvp isoform-specific RNAi. Newly emerged sixth instar female nymphs were injected with $3 \mu \mathrm{g}$ of $d s M o c k$ (Control), $d s B g S v p-A$ (BgSvp-Ai) or $d s B g S v p-B(B g S v p-B i)$. (A) mRNA levels of $B g S v p-A$ and $B g S v p-B$, relative to $B g$ Actin $5 C$ levels, in the CA of 6-day-old N6 specimens, measured by qRT-PCR. (B) DAPI-stained ovarioles from 5-day-old Control, $B g S v p$ - $A i$ and $B g S v p$ - $B i$ adult females. Arrowheads indicate basal oocytes. (C) $B g V g$ mRNA levels in the fat body of 5-day-old Control, BgSvp-Ai, and BgSvp-Bi females, relative to BgActin5C mRNA levels, measured by qRT-PCR. Error bars in $(\mathbf{A}$ and $\mathbf{C})$ represent SEM $(\mathrm{n}=5)$; asterisks in $(\mathbf{A}$ and $\mathbf{C})$ indicate differences statistically significant with respect to Controls $\left({ }^{* *} p \leq 0.0001\right.$ (Student's $t$ test)). Scale bar in (B): $200 \mu \mathrm{m}$.

One possibility emerging from the previous result is that the impairment of JH synthesis in $B g S v p-B i$ adults might be the consequence of the reduced number of CA cells. To test this, we depleted $B g S v p-B$ in the second adult gonadotrophic cycle when the final cell number in the adult CA has been already reached at the onset of the first gonadotrophic cycle. Thus, we injected $d s B g S v p-B$ and $d s$ Mock in females during the first day of ootheca transport, and then, the ootheca was removed 11 days later to induce the onset of the second gonadotrophic cycle (Fig. 8A). Under these conditions, none of the BgSvp-Bi animals $(\mathrm{n}=20)$ oviposited at the end of the second cycle. The detailed analysis of these animals revealed a complete block in egg development (Fig. 8B), and severely reduced levels of $B g V g$ mRNA in the fat body (Fig. 8C) and BgHMG-S1 and BgHMG-R in the CA (Fig. 8D). In contrast, the reduction of $B g S v p-B$ levels did not affect the mRNA levels of both enzymes in the fat body (Fig. 8E). Therefore, the results were similar to those obtained with specimens treated as nymphs and analyzed in the first gonadotrophic cycle, which indicate that the impairment in JH synthesis in $B g S v p-B i$ adults is not due to a reduced CA cell number but rather to the absence of BgSvp-B.

Nuclear receptor BgFTZ-F1 is present in CA cells during the adult period. Considering the tissue-specificity and the constant developmental expression of BgSvp-B in the CA, it seems likely that BgSvp-B is critical for the spatial control of JH biosynthetic gene expression in the CA but not for the temporal regulation during the adult period. Searching for regulatory factors involved in the temporal control of JH synthesis, we noticed that the timing of $B g H M G-S 1$ and $B g H M G-R$ up-regulation in the nymphal-adult transition correlates well with the decline of the $20 \mathrm{E}$ pulse that triggers such transition. We have previously demonstrated that a complex 20E-triggered hierarchy of nuclear receptors, converging in the strong up-regulation of $B g F T Z$ - F1 at the imaginal transition, regulates the imaginal molt ${ }^{32-37}$. Consistently, we found that this $20 \mathrm{E}$-induced cascade of nuclear receptors, composed by $B g E 75 A, B g H R 3-A, B g H R 4$, and BgFTZ-F1 was sequentially expressed also in last nymphal instar CA cells (Fig. 9A). Remarkably, the increase in BgFTZ-F1 mRNA levels during the last 2 days of the instar parallel those of BgHMG-S1 and BgHMG-R in this gland during the imaginal transition (Fig. 9A). Consistent with a possible role in the control of JH biosynthesis, BgFTZ-F1 was also expressed in the CA cells during the adult stage (Fig. 9B). However, the same changes in the mRNA levels of BgFTZ-F1 were also observed in the penultimate-to-last nymphal instar transition (Fig. 9B).

To assess whether BgFTZ-F1 could act as an adult-specific factor in the CA, we next analyzed the protein levels of BgFTZ-F1 in CA cells by an electrophoretic mobility shift assay (EMSA) analysis. To this aim, we first searched for a FTZ-F1 response element (F1RE) ${ }^{38}$ in the promoter/enhancer region of the BgHMG-S1 gene ${ }^{39}$. A detailed inspection revealed the presence of two potential F1REs, F1RE-S1a and F1RE-S1b (Supplementary Fig. S3). EMSA analysis utilizing BgFTZ-F1 protein translated in vitro and the F1RE-S1a element as a probe revealed a strong binding complex (Fig. 10A; lanes 2 and 3). In contrast, BgFTZ-F1 was not able to bind to the F1RE-S1b element as revealed by competition analysis (Fig. 10A; lane 4). The identity of the complex was confirmed with an antibody that specifically recognizes BgFTZ-F1 ${ }^{34}$ (Fig. 10A; lanes 5 and 6). Once demonstrated that in vitro-produced BgFTZ-F1 protein binds to the F1RE-S1a element, we examined the pattern of BgFTZ-F1 protein in CA nuclear extracts during the last two nymphal instars and the adult period. As a previous step, we characterized the occurrence of BgFTZ-F1 protein in nuclear extracts from CA from 5-day-old adult females, by incubating the extract with the F1RE-S1a element (Fig. 10B). We detected a retarded complex whose identity was confirmed with the BgFTZ-F1 antibody (Fig. 10B; lanes 1-3). The identity of the DNA-protein complex was further confirmed as the binding disappeared in CA extracts from $d s B g F T Z-F 1$-treated adults (Fig. 10B; lanes 4 and 5). 
A

A

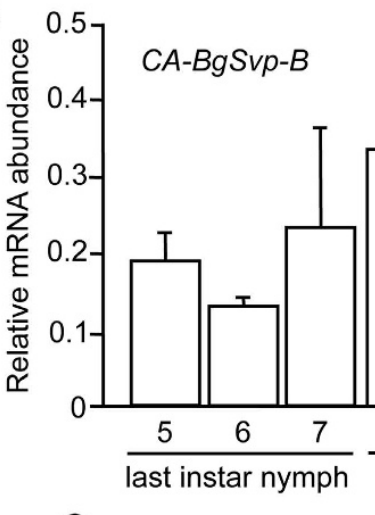

C

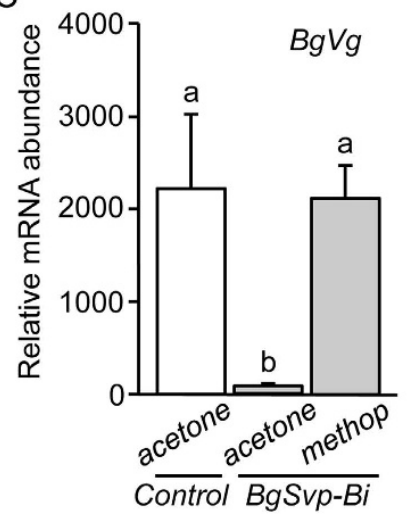

B

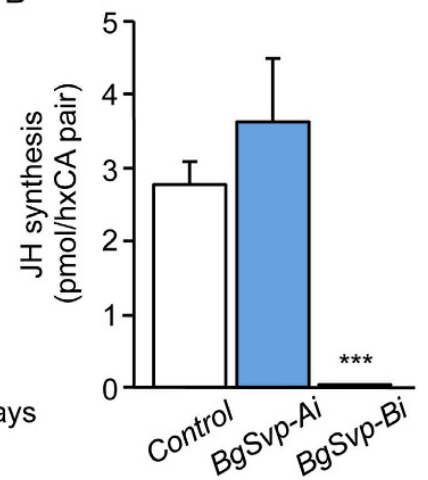

D

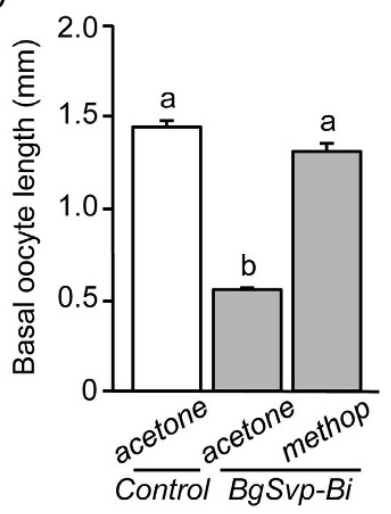

Figure 4. BgSvp-B controls JH production in B. germanica adult females. (A) Expression patterns of $B g S v p-B$, relative to BgActin5C levels, in the CA of B. germanica females during the last nymphal instar and the adult stage, measured by qRT-PCR. (B-D) Newly emerged sixth instar female nymphs were injected with $3 \mu \mathrm{g}$ of $d s B g S v p-A$ (BgSvp-Ai), $d s B g S v p-B$ (BgSvp-Bi) or with $d s M o c k$ (Control). (B) Rates of JH synthesis by CA incubated in vitro from 5-day-old Control, BgSvp-Ai and BgSvp-Bi adult females. (C) Expression levels of $B g V g$, relative to $B g A c t i n 5 C$ levels, in the fat body of 5-day-old $B g S v p$ - $B i$ adult females treated with acetone or methoprene, compared to acetone-treated Control adults, measured by qRT-PCR. (D) Basal oocyte length of 5-day-old BgSvp-Bi adult females treated with acetone or methoprene, compared to acetone-treated Control adults. Error bars represent SEM $(\mathrm{n}=3-10)$. Asterisks in $(\mathbf{B})$ indicate differences statistically significant with respect to Controls ( ${ }^{* *} p \leq 0.0001$; Student's $t$ test). Different letters in (C and $\left.\mathbf{D}\right)$ represent groups with significant differences according to ANOVA test (Tukey, $p \leq 0.005$ ).

Then, we tested the binding activity of BgFTZ-F1 in nuclear extracts from CA from staged penultimate and last nymphal instars and adult animals. Remarkably, BgFTZ-F1 binding activity was only detected in the imaginal transition and during the entire adult stage (Fig. 10C and D). Taken together, these findings show that BgFTZ-F1 protein competent to bind to the FTZ-F1 response element in the BgHMG-S1 gene is specifically present in the CA cells during the transition from the last nymph to adult and along the adult stage of B. germanica females, suggesting that it could act as an adult-specific transcription factor required for $\mathrm{JH}$ biosynthesis during this period.

BgFTZ-F1 is required for $\mathrm{JH}$ synthesis in adult $B$. germanica. To address the role of BgFTZ-F1 on JH synthesis, we next investigated the effect of $B g F T Z-F 1$ depletion during the adult stage by injecting $d s B g F T Z-F 1$ into newly molted last instar nymphs ( $B g F T Z-F 1 i$ animals). In agreement with our previous results [36], most of the BgFTZ-F1i nymphs $(75 \% ; \mathrm{n}=96)$ arrested development at the imaginal molt (Fig. 11A). However, $25 \%$ of $B g F T Z-F 1 i$ nymphs ecdysed properly into adults, with minor problems in the extension of the wings (Fig. 11A), thus allowing the analysis of BgFTZ-F1 functions during the adult stage. Similar to BgSvp- $B$, loss of $B g F T Z-F 1$ drastically impaired $\mathrm{JH}$ production in the CA, $B g V g$ expression in the fat body, and egg development (Fig. 11B-D). Likewise, mRNA levels of $B g H M G-S 1$ and $B g H M G-R$ in the CA of BgFTZ-F1 $i$ adults were significantly lower than those from Control animals (Fig. 11E). As a result of these deficiencies, BgFTZ-F1i adults did not oviposit nor produced any indication of ootheca formation at the end of the gonadotrophic cycle. Finally, to analyze whether BgSvp-B is involved in the control of BgFTZ-F1 expression in adult CA cells, we measured BgFTZ-F1 mRNA levels in the CA of $B g S v p-B i$ adults. As Fig. $11 \mathrm{~F}$ shows, mRNA levels of BgFTZ-F1 in the CA of $B g S v p-B i$ adults were not significantly affected, indicating that the role of BgSvp- $\mathrm{B}$ in JH biosynthesis is not channeled through the regulation of $B g F T Z-F 1$ expression in this gland. Similarly, we found that knocking down $B g F T Z-F 1$ did not affect $B g S v p-B$ expression levels in adult CA (Fig. 11G). Overall, our results show that BgFTZ-F1 is a critical factor in the adult-specific control of JH biosynthesis that acts in parallel to BgSvp-B. 
A

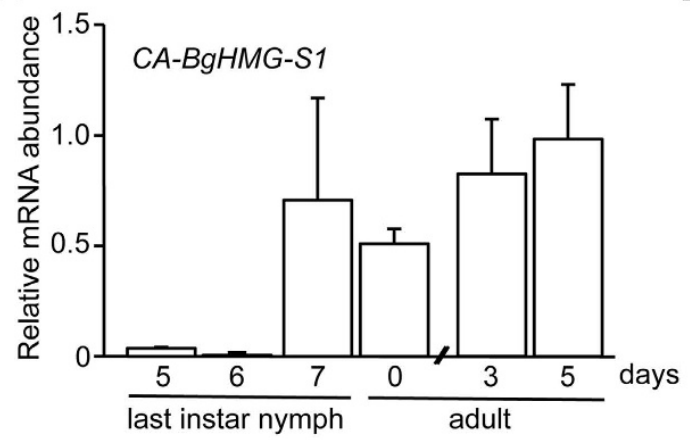

C

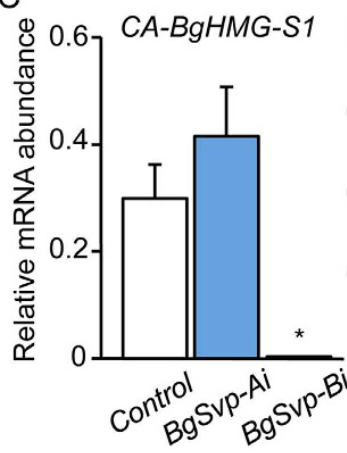

D

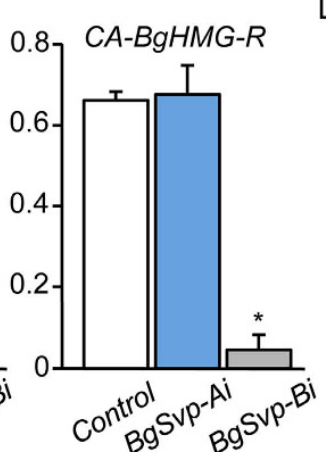

B
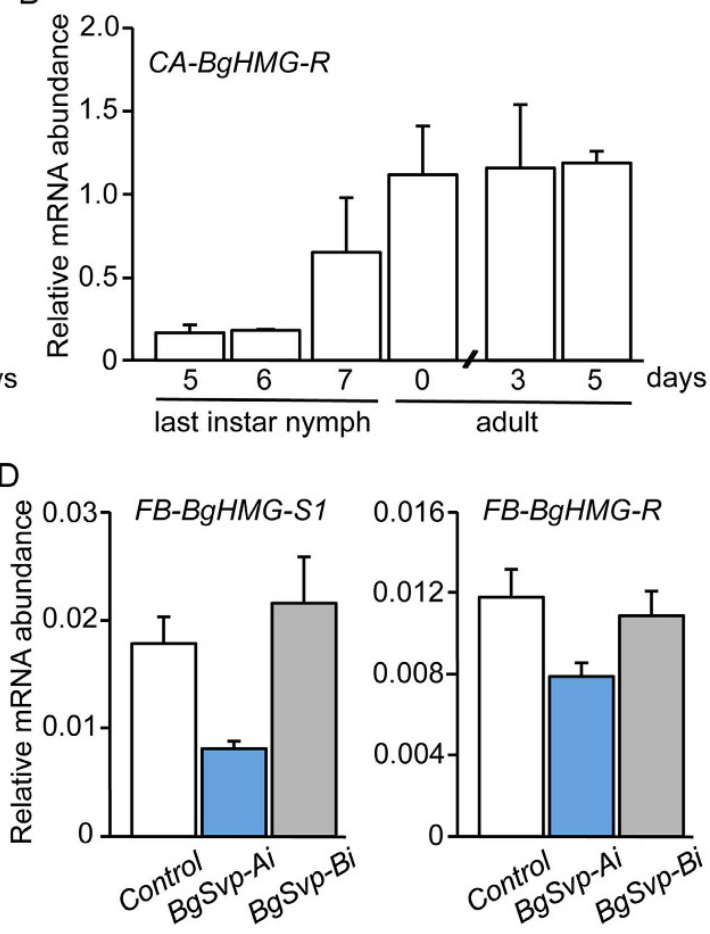

Figure 5. BgSvp-B controls the expression of JH biosynthetic enzyme genes in the CA of $B$. germanica adult females. (A and B) Expression patterns of $B g H M G-S 1$ (A) and $B g H M G-R$ (B), relative to $B g A c t i n 5 C$ levels, in the CA of B. germanica females during the last nymphal instar and the adult stage, measured by qRT-PCR. (C) BgHMG-S1 and BgHMG-R mRNA levels in the CA of 5-day-old Control, BgSvp-Ai and BgSvp-Bi females, relative to BgActin5C mRNA levels, measured by qRT-PCR. (D) BgHMG-S1 and BgHMG-R mRNA levels in the

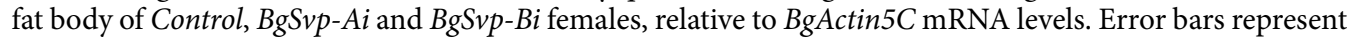
$\operatorname{SEM}(\mathrm{n}=6)$. Asterisks in $(\mathbf{C})$ indicate differences statistically significant with respect to Controls $\left({ }^{\star} p \leq 0.0001\right.$; Student's $t$ test).

A

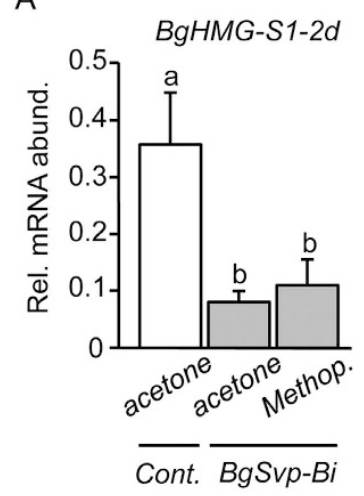

B

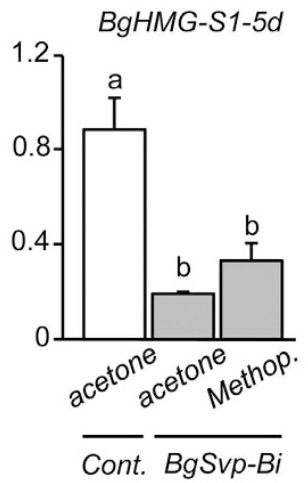

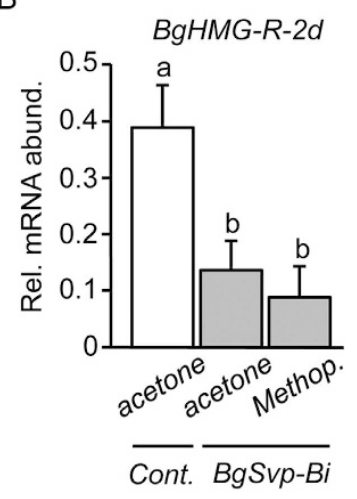

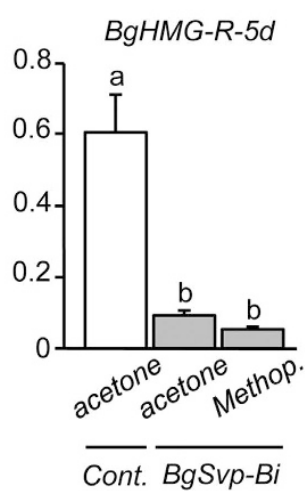

Figure 6. BgHMG-S1 and BgHMG-R reduction in CA of $B g S v p-B i$ animals is not due to the absence of JH. (A and B) Expression levels of BgHMG-S1 (A), and BgHMG-R (B), relative to BgActin5C levels, in the CA of 2 and 5-day-old $B g S v p$-Bi adult females treated with acetone or methoprene, compared to acetone-treated Control adults. Error bars represent SEM $(n=3-5)$. Different letters represent groups with significant differences according to ANOVA test (Tukey, $p \leq 0.05$ ).

\section{Discussion}

During insect development the levels of JH must be appropriately regulated to control the transition between the metamorphic and adult periods. Whereas the metamorphic stage is initiated by the inhibition of the JH biosynthetic activity of the CA cells, the onset of the reproductive adulthood is controlled by the precise re-induction of the CA activity at the imaginal molt ${ }^{4-7,40}$. This critical switch, therefore, constitutes an interesting paradigm for the study of the precise integration of tissue- and stage-specific signals required for the control of a basic developmental process. In the present study, we identify two transcription factors, BgSvp-B and BgFTZ-F1 that 


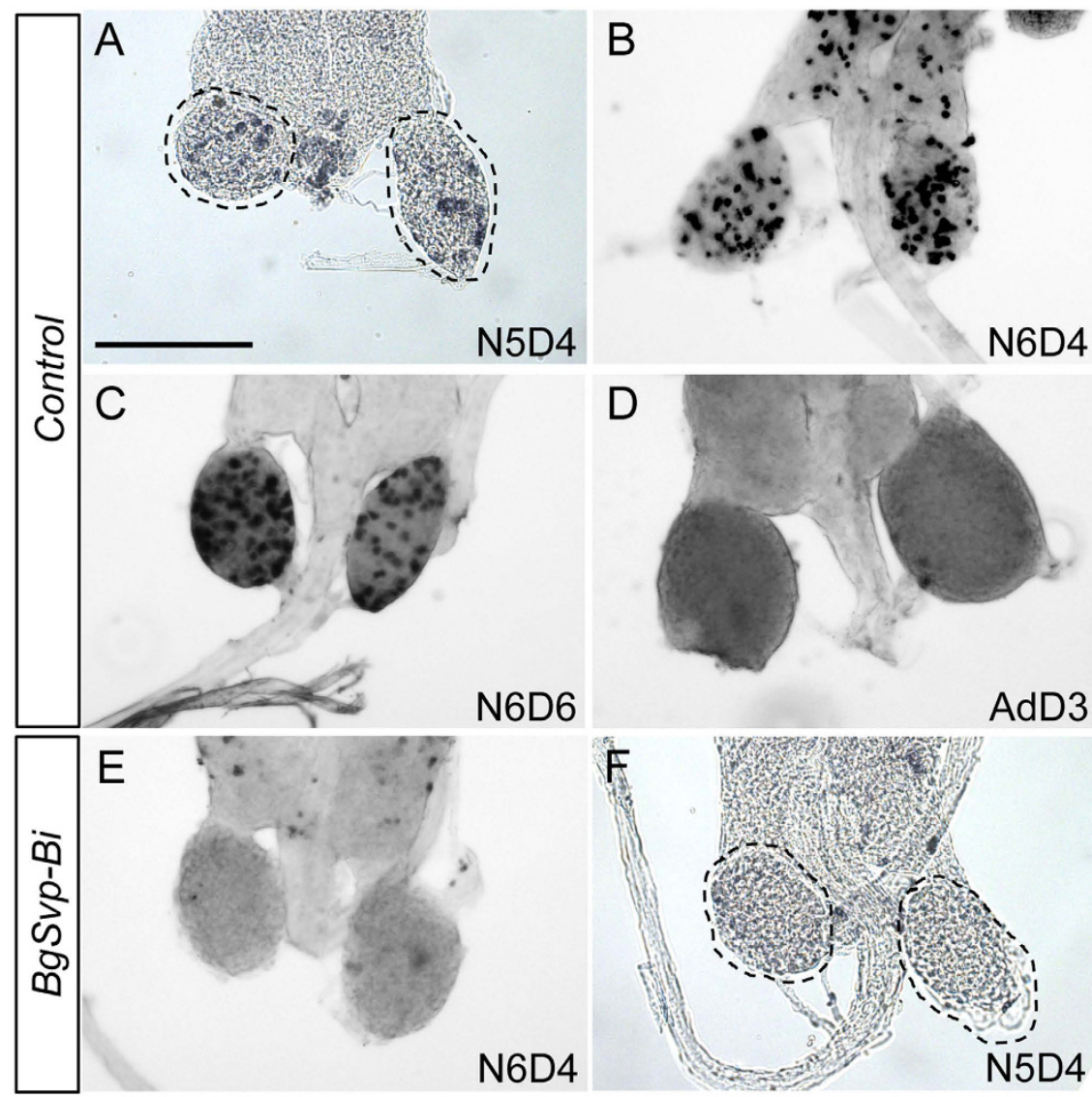

Figure 7. BgSvp-B promotes proliferation of CA cells during nymphal development in B. germanica. Control and BgSvp-Bi animals were pulsed with BrdU and the CA were dissected and stained to reveal BrdU incorporation. CA from penultimate (N5) (A), and last (N6) nymphal stages at the indicated ages (B and C) show abundant BrdU-labeled cells, while CA from adult females (D) show no BrdU incorporation. (E and F) CA from N6 and N5 BgSvp-Bi nymphs do not show any BrdU-labeled cells. Scale bar in (A): $200 \mu \mathrm{m}$.

are necessary to guarantee the strong activation of key JH biosynthetic enzymes in the CA cells required for the massive production of $\mathrm{JH}$ associated to adulthood.

BgSvp-B is critical for proper CA function. A key aspect of $\mathrm{JH}$ synthesis regulation is how the CA-specific expression of $\mathrm{JH}$ biosynthetic enzymes is controlled. Our work provides several lines of evidence demonstrating that the nuclear receptor BgSvp-B is a critical transcriptional regulator of JH biosynthetic enzymes in the CA of adult B. germanica. To our knowledge, this is the first case in which one isoform of Svp has been linked specifically to the spatial control of JH biosynthesis in insects. To date, Svp had been shown to control the correct development of the photoreceptor cells of the ommatidium ${ }^{41,42}$, the formation of the embryonic central and peripheral nervous systems, as well as the fat body, Malpighian tubules and the different cardioblasts in the dorsal vessel of D. melanogaster ${ }^{4-46}$. In the coleopteran Tribolium castaneum, TcSvp has been connected with the regulation of the metamorphic process ${ }^{47}$. However, the fact that $S v p$ is also highly expressed in the corpus allatum of D. melanogaster during early embryogenesis ${ }^{27}$, might suggest that this factor could play a conserved role in the transcriptional control of $\mathrm{JH}$ synthesis in insects. Consistent with this possibility, it has been recently shown that TcSvp is important for reproduction in adult females of $T$. castaneum, as revealed by the fact that newly eclosed T. castaneum female adults treated with $d s T c S v p$ show significant egg production impairment and reduced $T c V g$ mRNA levels in the fat body ${ }^{48}$.

In addition to the functions described, Svp also exerts regulatory functions in holometabolous insects through the attenuation of the activity of the 20E-signalling cascade by inhibiting the normal functioning of the EcR-USP heterodimer ${ }^{49,50}$. In A. aegypti, AaSvp interacts with AaUSP at the end of the vitellogenic cycle inhibiting the $20 \mathrm{E}$-dependent transactivation of target genes such as $A a V g^{51,52}$. Likewise, ectopic overexpression of Svp in larval and pupal stages of $D$. melanogaster leads to lethality at the onset of metamorphosis due to the impairment of the normal activity of the EcR-USP complex ${ }^{49}$. In B. germanica, we have shown that the same 20E-triggered hierarchy of nuclear receptors is expressed during nymphal development and controls the metamorphic process, including proper molting ${ }^{36}$. However, in contrast to holometabolous insects, $B g S v p$ isoforms seem not to be involved in $20 \mathrm{E}$-dependent processes as revealed by the fact that the BgSvp-depleted nymphs developed normally and reached adulthood without any noticeable defect. 
A

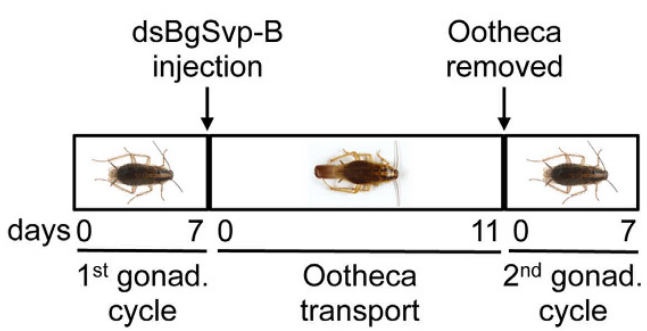

D
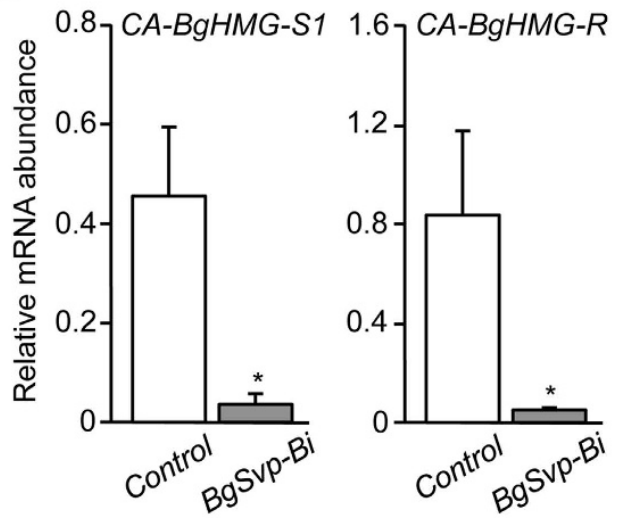

B

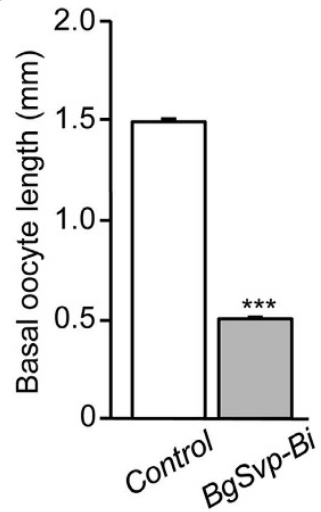

$\mathrm{E}$

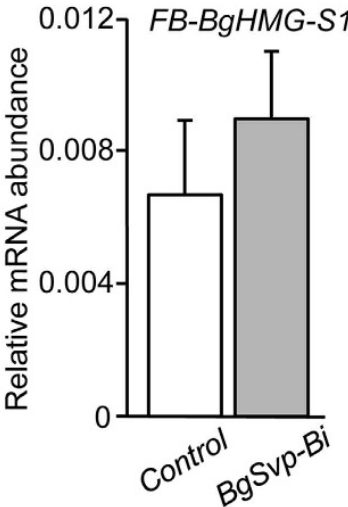

C

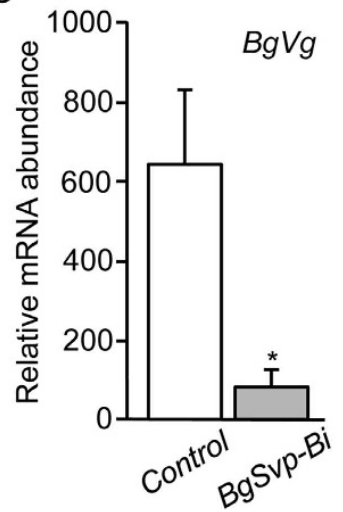

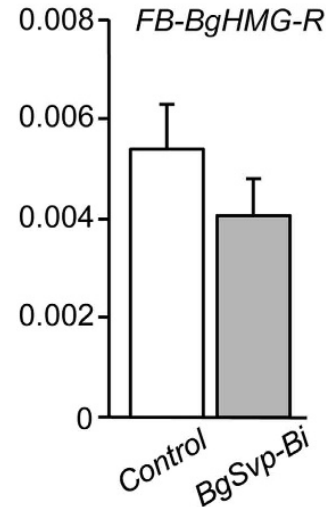

Figure 8. BgSvp-B controls JH production in the second gonadotrophic cycle of B. germanica. (A) Diagram of the experimental design to analyze the role of BgSvp-B in the second gonadotrophic cycle of adult B. germanica. (B) Basal oocyte length of 5-day-old Control and BgSvp- $1 i$ adult females. Results are expressed as the mean S.E. ( $\mathrm{n}=15-20)$. (C-E) Transcript levels of $B g V g$ in the fat body (C), BgHMG-S1 and BgHMG-R in the CA (D), and $B g H M G-S 1$ and $B g H M G-R$ in the fat body (E) of second gonadotrophic cycle 5-day-old Control and BgSvp-Bi adults, measured by qRT-PCR. Transcript abundance values are normalized against the BgActin $5 \mathrm{C}$ transcript. Error bars indicate the SEM $(\mathrm{n}=5-10)$. Asterisks indicate differences statistically significant ${ }^{*} p \leq 0.05 ;{ }^{* * *} p \leq 0.0005$, Student's $t$-test).

A

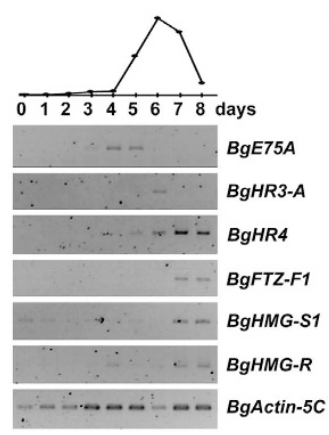

B

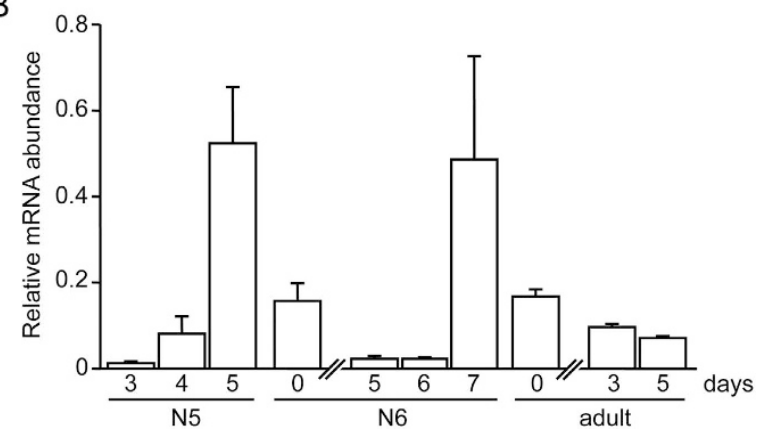

Figure 9. BgFTZ-F1 is expressed in the CA of B. germanica. (A) Expression patterns of 20-hydroxyecdysonedependent nuclear receptors during the last nymphal instar of $B$. germanica, analyzed by semi-quantitative RT-PCR. BgActin5C mRNA levels were used as a reference. The blot is representative of three replicates. 20-hydroxyecdysone levels (upper part) are redrawn from ref. 18. (B) Expression pattern of $B g F T Z$ - $F 1$, relative to BgActin $5 \mathrm{C}$ levels, in the CA of B. germanica females during the penultimate and last nymphal instars and the adult stage, measured by qRT-PCR.

It is important to note that, despite B. germanica having two isoforms that are highly expressed in the CA cells, the control of $\mathrm{JH}$ synthesis is exerted specifically by the BgSvp-B isoform. Both isoforms are identical except for the N-terminal end of the molecule. BgSvp-B presents a highly conserved 229 amino acid LBD with a canonical 
A

TNT BgFTZ-F1 -+++++
F1RE-S1a 100x - - + - -
F1RE-S1b 100x - - + + -
pre-imm. serum - - - +
anti-BgFTZ-F1 - - - - +

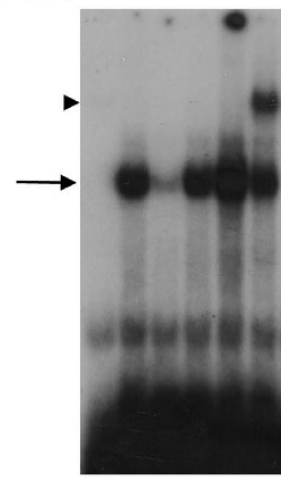

C
B

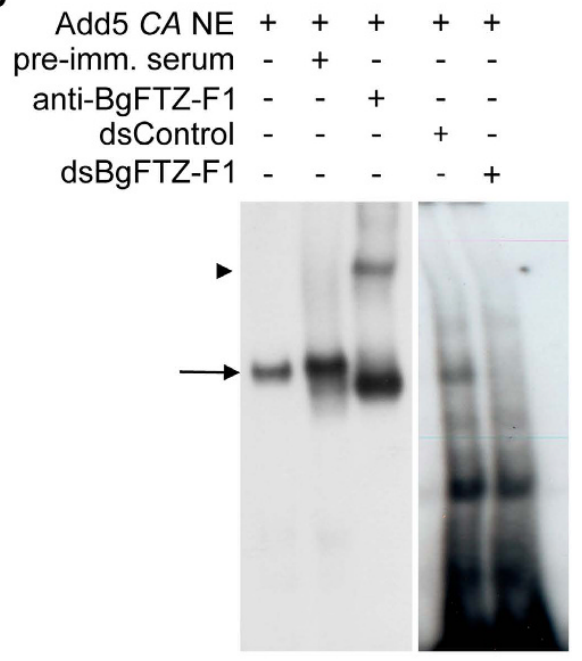

D

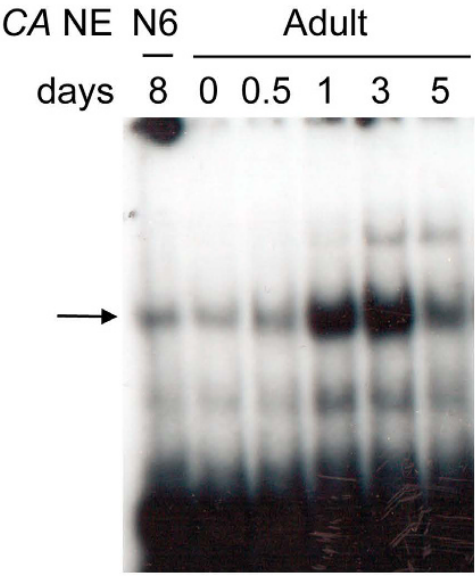

Figure 10. BgFTZ-F1 protein is present in the CA of $B$. germanica adult females. (A) EMSA analysis of the BgFTZ-F1 protein translated in vitro. The protein sample was incubated with ${ }^{32} \mathrm{P}-$ labeled F1RE-S1a response element. The specificity of the interaction was tested by the addition of 100 -fold molar excess of unlabeled F1RE-S1a and F1RE-S1b elements. The identity of the complex was tested with the addition of anti-BgFTZ-F1 antibody (anti-BgFTZ-F1) and pre-immune serum (pre-imm. serum). (B) Identification of BgFTZ-F1 protein in CA nuclear extracts (CA NE) of B. germanica 5-day-old adult females. The identity of the complex was tested with the addition of anti-BgFTZ-F1 and pre-imm serum, as well as with the analysis of CA NE from $d s B g F T Z$ F1-treated adults from the same age. (C) Developmental profile of BgFTZ-F1 protein present in CA NE from penultimate (N5) and last (N6) instar nymphs, as well as adult females incubated with labeled F1RE-S1a element. 20 CA equivalent was used in each binding reaction. (D) Developmental profile of BgFTZ-F1 protein present in CA NE from females of the first gonadotrophic cycle incubated with labeled F1RE-S1a element. Arrows in (A-D) indicate the BgFTZ-F1-F1RE-S1a complex. Arrowheads in (A and B) indicate supershifted BgFTZ-F1-F1RE-S1a complexes due to the addition of anti-BgFTZ-F1.

structure formed by $12 \alpha$-helices, including the ligand-dependent transactivation domain (AF-2) within $\alpha$-helix 12 , which has been shown to be responsible for the interaction with different coactivator and corepressor proteins $^{53}$. In contrast, BgSvp-A presents a truncated 139 amino acid LBD, with the last 21 amino acids presenting no homology with any other member of the nuclear receptor superfamily. Remarkably, the truncated LBD of BgSvp-A lacks the last $4 \alpha$-helices, including the AF- 2 domain, which suggests that this region of the protein is key to exert the regulatory functions upon JH synthesis. Further studies are needed to establish the role of the carboxy-end of the LBD in the control of JH biosynthesis by BgSvp-B.

In cockroaches, including B. germanica, CA cells proliferate continuously during nymphal development and switch to an adult-specific non-proliferative state at the imaginal molt concomitantly to the increase of JH production (Fig. 7A-D) ${ }^{24,25}$. Here, we have shown that the CA cells require the continuous activity of BgSvp-B during nymphal development to maintain their proliferative capacity as revealed by BrdU experiments. Svp has likewise been implicated in the control of cell proliferation in the Malpighian tubules in D. melanogaster through the induction of the expression of two cell cycle regulators, the cdc23 phosphatase string and cyclinE ${ }^{44}$, thus suggesting that the regulation of cell proliferation by Svp is conserved in different insect tissues. Overall, our results show 
A

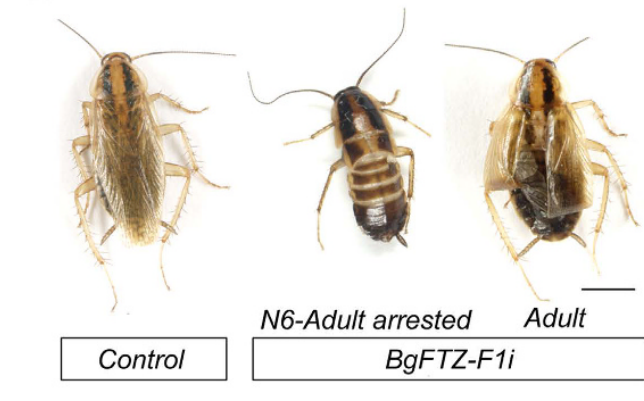

B

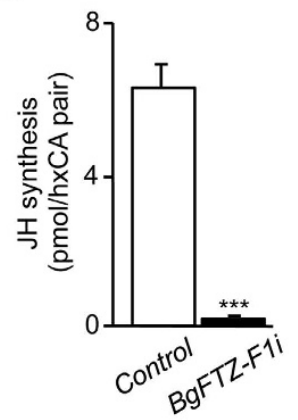

C

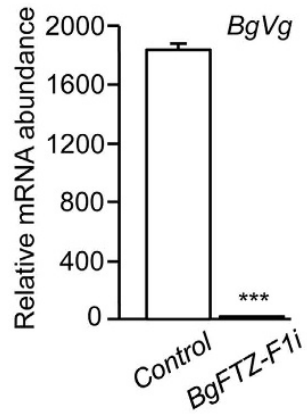

G

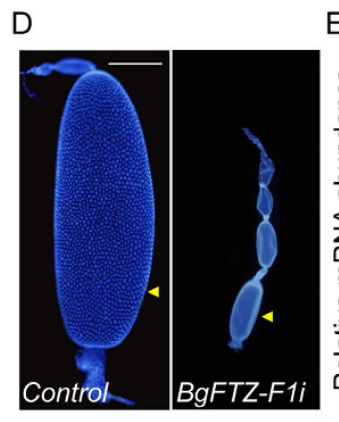

E

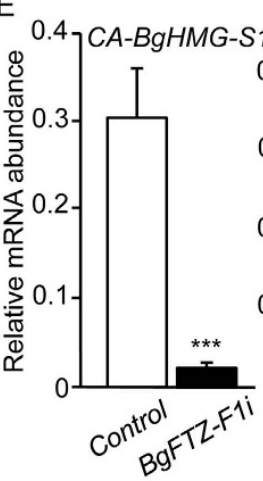

$C A-B g H M G-R$ $\mathrm{F}$
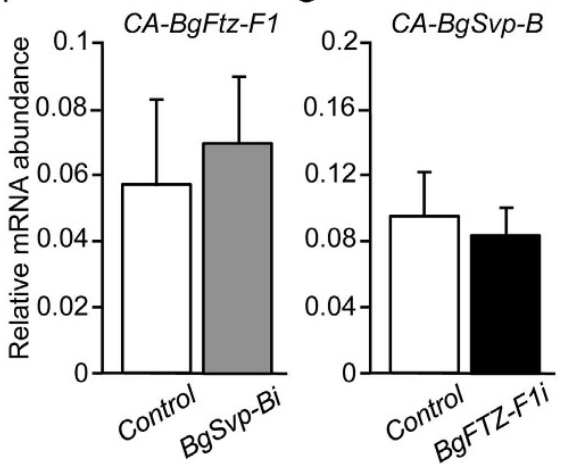

Figure 11. Loss of BgFTZ-F1 impairs JH synthesis in B. germanica adult females. (A) Newly emerged sixth instar female nymphs were injected with $3 \mu \mathrm{g}$ of $d s M o c k$ (Control) or $d s B g F T Z-F 1$ (BgFTZ-F1i). Dorsal views of Control and BgFTZ-F1i animals. BgFTZ-F1i animals either arrested development at the transition between the last nymphal instar and the adult stage (left) or molted properly into adults with only minor problems in the extension of the wings (right). (B) Rates of JH synthesis by CA incubated in vitro from 5-day-old Control and $B g F T Z-F 1 i$ adult females. (C) BgVg mRNA levels in the fat body of 5-day-old Control and BgFTZ-F1i females, relative to BgActin5C mRNA levels, measured by qRT-PCR. (D) DAPI-stained ovarioles from 5-day-old Control and BgFTZ-F1i adult females. Arrowheads indicate basal oocytes. (E) BgHMG-S1 and BgHMG-R mRNA levels in the CA of 5-day-old Control and BgFTZ-F1i females, relative to BgActin5C mRNA levels, measured by qRTPCR. (F) BgFTZ-F1 mRNA levels in the CA of 5-day-old Control and BgSvp-Bi females, relative to BgActin5C mRNA levels, measured by qRT-PCR. (G) BgSvp-B mRNA levels in the CA of 5-day-old Control and BgFTZ-F1i females, relative to BgActin5C mRNA levels, measured by qRT-PCR. Error bars indicate the SEM $(\mathrm{n}=3-10)$. Asterisks indicate differences statistically significant ${ }^{* * *} p \leq 0.0005$, Student's $t$-test). Scale bar in (D): $1 \mathrm{~mm}$.

that BgSvp-B is a critical factor that exerts a dual role in the control of the CA in B. germanica. First, controlling cell proliferation during the successive nymphal stages, and second, promoting the massive production of $\mathrm{JH}$ during the adult period by up-regulating $B g H M G-S 1$ and $B g H M G-R$ expression in the CA cells.

BgFTZ-F1 is a temporal regulator of JH synthesis. The constant expression of $B g S v p-B$ in the CA cells does not correlate with the strong up-regulation of $B g H M G-S 1$ and $B g H M G-R$ and the increase in JH production during the adult period, thus suggesting that other factors must act coordinately to ensure the adult-specific up-regulation of JH biosynthetic enzymes. In this regard, we have identified the nuclear receptor BgFTZ-F1 as responsible for such adult-specificity. Several results support this observation: (1) the binding levels of BgFTZ-F1 in the CA cells correlate well with the expression of BgHMG-S1 and BgHMG-R during the imaginal transition and the adult stage; (2) JH synthesis, $B g V g$ induction and oocyte growth are completely impaired in $B g F T Z-F 1 i$ animals; and (3) the mRNA levels of BgHMG-S1 and BgHMG-R are strongly reduced in the CA cells of BgFTZ-F1i adults.

It is interesting to note that a strong up-regulation of $B g F T Z-F 1$ at the imaginal molt is also observed in the prothoracic gland, the tissue responsible of the synthesis of ecdysteroid hormones ${ }^{34}$. In this case, $B g F T Z-F 1$ induces the degeneration of the prothoracic gland immediately after the imaginal molt, a critical event required for proper adult development in B. germanica ${ }^{54}$. BgFTZ-F1 acts, therefore, as a critical adult-specific factor in the two main endocrine glands, the prothoracic gland where induces its degeneration, and the CA where promotes the synthesis of high levels of JH. The role of FTZ-F1 as a stage-specific determinant has been previously demonstrated in the prepupal stage of D. melanogaster. In this particular period of development, $\beta$ FTZ-F1 works as a competence factor for stage-specific responses to the $20 \mathrm{E}$ pulse that triggers pupal development ${ }^{55}$. Similarly, in A. aegypti, $\beta \mathrm{FTZ}-\mathrm{F} 1$ is required for the stage-specific up-regulation of the 20E-dependent genes $E 74 B, E 75 \mathrm{~A}, \mathrm{Vg}$ and vitellogenic carboxypeptidase during the transition to the vitellogenic period ${ }^{51,52,56}$. 
In light of our results, in conclusion, we propose that the high level of $\mathrm{JH}$ biosynthesis required during the adult stage of B. germanica is achieved by the integration of tissular (BgSvp-B) and temporal (BgFTZ-F1) regulatory inputs. These inputs specifically converge in the CA cells at the imaginal transition and the adult period to guarantee the strong up-regulation of, at least, two important JH biosynthesis enzyme genes, $B g H M G-S 1$ and $B g H M G-R$. The present work, thus, represents a significant step toward understanding the molecular mechanisms underlying developmental progression in insects by deciphering how the stage- and tissue-specific responses are reached during development.

\section{Materials and Methods}

Insects. Specimens of B. germanica were obtained from a colony reared in the dark at $30 \pm 1{ }^{\circ} \mathrm{C}$ and $60-70 \%$ r.h. All dissection and tissue sampling were carried out in Ringer's saline using carbon dioxide-anesthetized specimens.

Cloning of BgSvp cDNAs. Degenerate primers based on the DNA binding domain (DBD) of Svp insect homologs were used to obtain a B. germanica homolog cDNA fragment by RT-PCR: forward primer (BgSvp-F1): 5'-AUHGARTGYGTNGTNTGY-3', and reverse primer (BgSvp-R1): 5'-NCCNVMYCANSRYTANGT-3'. The first amplification was carried out using as a template cDNA generated by reverse transcription from polyA $^{+}$RNA from 20E-treated UM-BGE-1 cells (derived from early embryos of B. germanica), as previously described ${ }^{32,57}$. The primers used can be found at S1 Table. The amplified fragment (120 bp) was subcloned into the pSTBlue-1 vector (Novagen) and sequenced. This was followed by $5^{\prime}$ and $3^{\prime}$ RACE ( $5^{\prime}$ - and $3^{\prime}$-RACE System Version 2.0; Invitrogen) to complete the sequence. For $5^{\prime}$-RACE, reverse primer was (BgSvp-R2): 5'-TGAGATTTCTCCTGACACTCCTCT-3'; and for 3'-RACE, forward primer was (BgSvp-F2): $5^{\prime}$-AGAGGAGTGTCAGGAGAAATCTCA-3'. All PCR products were subcloned into the pSTBlue-1 vector (Novagen) and sequenced in both directions. Following this approach, we isolated two different BgSvp isoforms.

Semiquantitative reverse transcriptase polymerase chain reaction (RT-PCR). RT-PCR was used to determine the expression pattern of BgSvp isoforms. Total RNA was extracted from tissues using the GenElute $^{\mathrm{TM}}$ Mammalian Total RNA kit (Sigma). cDNA synthesis was carried out as previously described ${ }^{18}$. Primers for the amplification of BgSvp isoforms and the different ecdysone-dependent nuclear receptors can be found at Supplementary Table S1. As a reference the same cDNAs were subjected to RT-PCR with a primer pair specific for B. germanica Actin5C as described ${ }^{57}$. cDNA samples were subjected to PCR with a number of cycles within the linear range of amplification for each transcript depending on the tissue and physiological stage.

Quantitative real-time reverse transcriptase polymerase chain reaction (qRT-PCR). Total RNA extraction and cDNA synthesis was carried out as described above. Relative transcripts levels were determined by quantitative real-time PCR (qPCR), using iQ SYBR Green supermix (Bio-Rad), in a $20 \mu$ l final volume (see Supplementary Table S2 for primer sequences). The qPCR experiments were conducted with the same quantity of organ equivalent input for all treatments and each sample was run in duplicate using $2 \mu \mathrm{l}$ of cDNA per reaction. All the samples were analyzed using an iCycler and iQ Real Time PCR Detection System (Bio-Rad). For each standard curve, one reference DNA sample was diluted serially.

RNA interference in vivo. RNAi in vivo in nymphs of B. germanica was performed as previously described $^{32,37}$. The primers used to generate templates via PCR for transcription of the dsRNAs are described in Supplementary Table S3. A volume of $1 \mu \mathrm{l}$ of each dsRNA solution $(3 \mu \mathrm{g} / \mu \mathrm{l})$ was injected into the abdomen of newly emerged female nymphs. To analyze the effect of the interference in the second adult gonadotrophic cycle, dsRNAs were injected into females in the first day of ootheca transport right after the end of the first gonadotrophic cycle. Eleven days after the injection, oothecas were removed to trigger the onset of the second gonadotrophic cycle.

Incubation of CA and quantification of juvenile hormone synthesis. JH III biosynthesis by CA incubated in vitro was quantified using the methodology previously reported ${ }^{30}$. Basically, individual corpora cardiaca-CA complexes were incubated for 3 hours in $100 \mu$ of 199 medium (Sigma) containing L-methionine $(0.1 \mathrm{mM})$, Hank's salts, HEPES $(20 \mathrm{mM})$ plus Ficoll $(20 \mathrm{mg} / \mathrm{ml})$, to which L- $\left[{ }^{3} \mathrm{H}\right.$-methyl $]$ methionine (Perkin Elmer) had been added to achieve a final specific activity of $7.4 \mathrm{Gbq} / \mathrm{mmol}$. After the incubation period JH III in the medium plus homogenized glands was extracted and quantified.

Treatments with methoprene in vivo. Newly ecdysed adults were topically treated with $1 \mu \mathrm{g}$ methoprene (isopropyl(E,E)-(RS)-11-methoxy-3,7,11-trimethyldodeca-2,4-dienoate) per specimen in $1 \mu l$ of acetone. Controls received the same volume of solvent.

Microscopy, histological analysis and protein electrophoresis. After dissection, ovaries were fixed in $4 \%$ paraformaldehyde and permeabilised in PBS- $0,2 \%$ Tween (PBT), then incubated for $10 \mathrm{~min}$ in $1 \mu \mathrm{g} / \mathrm{ml}$ DAPI in PBT. After two washes with PBT, the tissues were mounted in Mowiol 4-88 (Calbiochem). All samples were examined with a Zeiss Axiophot microscope, and images were subsequently processed using Adobe photoshop.

Proliferation of CA cells was monitored by in vivo labeling with $5^{\prime}$-bromo-2-deoxyuridine (BrdU). Nymph and adult insects were injected with a BrdU solution and $24 \mathrm{~h}$ later, CA were dissected and fixed in Carnoy's fixative for $30 \mathrm{~min}$, washed in PBS and incubated in $70 \%$ methanol $(\mathrm{MeOH})$ for $10 \mathrm{~min}, \mathrm{MeOH}+30 \%$ hydrogen peroxide $\left(\mathrm{H}_{2} \mathrm{O}_{2}\right)(1: 1)$ for $45 \mathrm{~min}$ and $70 \% \mathrm{MeOH}$ for $10 \mathrm{~min}$. CA were washed in PBS, and then incubated $1 \mathrm{~h}$ in PBST-BSA. They were incubated with $2 \mathrm{~N} \mathrm{HCl}$ for $30 \mathrm{~min}$ to denature the DNA and allow access to the anti-BrdU 
antibody. The tissue was then washed $3 \times 10 \mathrm{~min}$ in PBS and $2 \times 10 \mathrm{~min}$ PBST-BSA and placed in PBST-BSA-NGS blocking solution for $30 \mathrm{~min}$. Mouse anti-BrdU (Hybridoma bank) was added at a 1:1000 concentration to a PBST-BSA-NGS solution overnight at $4{ }^{\circ} \mathrm{C}$. Next, the tissues were washed $3 \times 10 \mathrm{~min}$ PBST-BSA and incubated $30 \mathrm{~min}$ in PBST-BSA-NSG prior to $2 \mathrm{~h}$ incubation with a peroxidase-labelled anti-mouse IgG secondary antibody (1:100 in PBS-BSA-NGS). Finally, the tissues were washed $3 \times 20 \mathrm{~min}$ PBS and immunoreactive cells were visualized by incubation in a solution of 3,3'-diaminobenezidine in PBS containing $\mathrm{H}_{2} \mathrm{O}_{2}$ and nickel chloride. Finally, CA cells were examined with Zeiss Axiophot microscope. SDS-PAGE of hemolymph and ovarian proteins was carried out as previously reported ${ }^{21}$.

In vitro transcription/translation. The BgFTZ-F1 cDNA was cloned into pSTBlue-1 (Novagen) ${ }^{34}$, and transcribed and translated using the TNT coupled reticulocyte lysate system (Promega), according to the manufacturer's instructions.

CA Nuclear extracts and EMSA. Preparations of nuclear extracts from B. germanica CA were carried out according to the method described ${ }^{58}$. Twenty CA were used for each time-point extraction. Binding reactions were carried out in a $20 \mu \mathrm{l}$ volume containing 20 CA equivalent of nuclear extracts or $1 \mu \mathrm{l}$ of the TNT sample, $10 \mathrm{mM}$ Tris- $\mathrm{HCl}$ (pH 7.5), $50 \mathrm{mM} \mathrm{NaCl}, 1 \mathrm{mMMgCl} 2,0.5 \mathrm{mM}$ DTT, $0.5 \mathrm{mM}$ EDTA, $4 \%$ (v/v) glycerol, $1 \mu \mathrm{g}$ poly(dI.dC), $1 \mu \mathrm{g}$ of a single-stranded DNA (5'-TAATACGACTCACTATA-3'), and the indicated amount of competitor DNA or antibody when appropriate. After $15 \mathrm{~min}$ incubating at $4{ }^{\circ} \mathrm{C}, 0.05 \mathrm{pmol}$ of ${ }^{32} \mathrm{P}$-labeled DNA probe was added, and the incubation was continued for another $45 \mathrm{~min}$ at the same temperature. The reaction was resolved on $5 \%$ nondenaturing polyacrilamide gel run at $4^{\circ} \mathrm{C}$ and at a constant voltage of $150 \mathrm{~V}$ in $0.5 \mathrm{X}$ TBE. The gel was then dried and autoradiographed. Oligonucleotides (only sense strands are shown) used to generate DNA probes for EMSA were: F1RE-S1a: 5'-GTTCAATTTGTTGACCGAAGGCCGCTATGTTTTCATCC-3'; F1RE-S1b: 5'-GAGTAATAGCCCTAGCCTTAAATTAACATGGGGCC-3'.

\section{References}

1. Jindra, M., Palli, S. R. \& Riddiford, L. M. The Juvenile Hormone Signaling Pathway in Insect Development. Annu. Rev. Entomol. 58, 181-204 (2013)

2. Riddiford, L. M. How does juvenile hormone control insect metamorphosis and reproduction? Gen. Comp. Endocrinol. 179, 477-84 (2012).

3. Marek, T., Xavier, Ji. \& Shinoda, B. Molecular basis of juvenile hormone signaling. Curr. Opin. Insect Sci. 11, 39-46 (2015).

4. Truman, J. W. \& Riddiford, L. M. The morphostatic actions of juvenile hormone. Insect Biochem. Mol. Biol. 37, 761-70 (2007)

5. Raikhel, A. S., Brown, M. R. \& Belles, X. In Comprehensive Molecular Insect Science (ed. Gilbert, L. I.) 433-491 (Elsevier, 2005).

6. Sheng, Z., Xu, J., Bai, H., Zhu, F. \& Palli, S. R. Juvenile hormone regulates vitellogenin gene expression through insulin-like peptide signaling pathway in the red flour beetle, Tribolium castaneum. J. Biol. Chem. 286, 41924-41936 (2011).

7. Comas, D., Piulachs, M. D. \& Belles, X. Fast induction of vitellogenin gene expression by juvenile hormone III in the cockroach Blattella germanica (L.) (Dictyoptera, Blattellidae). Insect Biochem. Mol. Biol. 29, 821-827 (1999).

8. Bellés, X., Martín, D. \& Piulachs, M.-D. The mevalonate pathway and the synthesis of juvenile hormone in insects. Annu. Rev. Entomol. 50, 181-199 (2005).

9. Kinjoh, T. et al. Control of juvenile hormone biosynthesis in Bombyx mori: Cloning of the enzymes in the mevalonate pathway and assessment of their developmental expression in the corpora allata. Insect Biochem. Mol. Biol. 37, 808-818 (2007).

10. Shinoda, T. \& Itoyama, K. Juvenile hormone acid methyltransferase: a key regulatory enzyme for insect metamorphosis. Proc. Natl. Acad. Sci. USA 100, 11986-91 (2003).

11. Nouzova, M., Edwards, M. J., Mayoral, J. G. \& Noriega, F. G. A coordinated expression of biosynthetic enzymes controls the flux of juvenile hormone precursors in the corpora allata of mosquitoes. Insect Biochem. Mol. Biol. 41, 660-9 (2011).

12. Rivera-Perez, C., Nouzova, M., Lamboglia, I. \& Noriega, F. G. Metabolic analysis reveals changes in the mevalonate and juvenile hormone synthesis pathways linked to the mosquito reproductive physiology. Insect Biochem. Mol. Biol. 51, 1-9 (2014).

13. Huang, J., Marchal, E., Hult, E. F. \& Tobe, S. S. Characterization of the juvenile hormone pathway in the viviparous cockroach, Diploptera punctata. PLoS One 10, e0117291 (2015).

14. Huang, J. et al. DPP-mediated TGFbeta signaling regulates juvenile hormone biosynthesis by activating the expression of juvenile hormone acid methyltransferase. Development 138, 2283-2291 (2011).

15. Cheng, C. et al. The POU factor ventral veins lacking/Drifter directs the timing of metamorphosis through ecdysteroid and juvenile hormone signaling. PLoS Genet. 10, e1004425 (2014).

16. Hiruma, K. \& Kaneko, Y. Hormonal regulation of insect metamorphosis with special reference to juvenile hormone biosynthesis. Curr. Top. Dev. Biol. 103, 73-100 (2013).

17. Camps, F., Casas, J., Sánchez, F. J. \& Messeguer, A. Identification of juvenile hormone III in the hemolymph of Blattella germanica adult females by gas chromatography-mass spectrometry. Arch. Insect Biochem. Physiol. 6, 181-189 (1987).

18. Cruz, J. et al. Quantity does matter. Juvenile hormone and the onset of vitellogenesis in the German cockroach. Insect Biochem. Mol. Biol. 33, 1219-1225 (2003).

19. Treiblmayr, K., Pascual, N., Piulachs, M.-D., Keller, T. \& Bellés, X. Juvenile hormone titer versus juvenile hormone synthesis in female nymphs and adults of the German cockroach, Blattella germanica. J. Insect Sci. 6, 1-7 (2006).

20. Comas, D., Piulachs, M. D. \& Belles, X. Induction of vitellogenin gene transcription in vitro by juvenile hormone in Blattella germanica. Mol. Cell. Endocrinol. 183, 93-100 (2001).

21. Martín, D., Piulachs, M.-D. \& Bellés, X. Patterns of haemolymph vitellogenin and ovarian vitellin in the German cockroach, and the role of Juvenile Hormone. Physiol. Entomol. 20, 59-65 (1995).

22. Martín, D., Piulachs, M.-D. \& Bellés, X. Production of vitellogenin in vitro by the periovaric fat body of Blattella germanica(L.) (Dictyoptera, Blattellidae). Invertebr. Reprod. \& Dev. 28, 171-176 (1995).

23. Martin, D., Piulachs, M. D., Comas, D. \& Belles, X. Isolation and sequence of a partial vitellogenin cDNA from the cockroach, Blattella germanica (L.) (Dictyoptera, Blattellidae), and characterization of the vitellogenin gene expression. Arch. Insect Biochem. Physiol. 38, 137-146 (1998).

24. Chiang, A. S., Gadot, M., Burns, E. L. \& Schal, C. Sexual differentiation of nymphal corpora allata and the effects of ovariectomy on adult gland morphometrics in Blattella germanica. Experientia 47, 81-83 (1991).

25. Urena, E., Manjon, C., Franch-Marro, X. \& Martin, D. Transcription factor E93 specifies adult metamorphosis in hemimetabolous and holometabolous insects. Proc. Natl. Acad. Sci. 201401478, doi: 10.1073/pnas.1401478111 (2014).

26. Ryan, K. M., Hoshizaki, D. K. \& Cripps, R. M. Homeotic selector genes control the patterning of seven-up expressing cells in the Drosophila dorsal vessel. Mech. Dev. 122, 1023-1033 (2005). 
27. Sánchez-Higueras, C., Sotillos, S. \& Castelli-Gair Hombría, J. Common origin of insect trachea and endocrine organs from a segmentally repeated precursor. Curr. Biol. 24, 76-81 (2014).

28. Martinez-Gonzalez, J., Buesa, C., Piulachs, M. D., Belles, X. \& Hegardt, F. G. 3-Hydroxy-3-methylglutaryl-coenzyme-A synthase from Blattella germanica. Cloning, expression, developmental pattern and tissue expression. Eur. J. Biochem. 217, 691-699 (1993).

29. Martinez-Gonzalez, J., Buesa, C., Piulachs, M. D., Belles, X. \& Hegardt, F. G. Molecular cloning, developmental pattern and tissue expression of 3-hydroxy-3-methylglutaryl coenzyme A reductase of the cockroach Blattella germanica. Eur. J. Biochem. 213, 233-241 (1993).

30. Maestro, J. L., Cobo, J. \& Bellés, X. Target of rapamycin (TOR) mediates the transduction of nutritional signals into juvenile hormone production. J. Biol. Chem. 284, 5506-5513 (2009).

31. Abrisqueta, M., Suren-Castillo, S. \& Maestro, J. L. Insulin receptor-mediated nutritional signalling regulates juvenile hormone biosynthesis and vitellogenin production in the German cockroach. Insect Biochem. Mol. Biol. 49, 14-23 (2014).

32. Cruz, J., Mané-Padrós, D., Bellés, X. \& Martín, D. Functions of the ecdysone receptor isoform-A in the hemimetabolous insect Blattella germanica revealed by systemic RNAi in vivo. Dev. Biol. 297, 158-171 (2006).

33. Cruz, J., Martín, D. \& Bellés, X. Redundant ecdysis regulatory functions of three nuclear receptor HR3 isoforms in the directdeveloping insect Blattella germanica. Mech. Dev. 124, 180-189 (2007).

34. Cruz, J., Nieva, C., Mané-Padrós, D., Martín, D. \& Bellés, X. Nuclear receptor BgFTZ-F1 regulates molting and the timing of ecdysteroid production during nymphal development in the hemimetabolous insect Blattella germanica. Dev. Dyn. 237, 3179-3191 (2008).

35. Mané-Padrós, D. et al. The nuclear hormone receptor BgE75 links molting and developmental progression in the direct-developing insect Blattella germanica. Dev. Biol. 315, 147-160 (2008).

36. Mane-Padros, D., Borràs-Castells, F., Bellés, X. \& Martín, D. Nuclear receptor HR4 plays an essential role in the ecdysteroidtriggered gene cascade in the development of the hemimetabolous insect Blattella germanica. Mol. Cell. Endocrinol. 1-9, doi: 10.1016/j.mce.2011.09.025 (2011).

37. Martín, D., Maestro, O., Cruz, J., Mané-Padrós, D. \& Bellés, X. RNAi studies reveal a conserved role for RXR in molting in the cockroach Blattella germanica. J. Insect Physiol. 52, 410-416 (2006).

38. Ohno, C. K., Ueda, H. \& Petkovich, M. The Drosophila nuclear receptors FTZ-F1 alpha and FTZ-F1 beta compete as monomers for binding to a site in the fushi tarazu gene. Mol. Cell. Biol. 14, 3166-75 (1994).

39. Casals, N., Buesa, C., Marrero, P. F., Belles, X. \& Hegardt, F. G. 3-Hydroxy-3-methylglutaryl coenzyme A synthase-1 of Blattella germanica has structural and functional features of an active retrogene. Insect Biochem. Mol. Biol. 31, 425-33 (2001).

40. Riddiford, L. M. How does juvenile hormone control insect metamorphosis and reproduction? Gen. Comp. Endocrinol. 179, 477-484 (2012).

41. Mlodzik, M., Hiromi, Y., Weber, U., Goodman, C. S. \& Rubin, G. M. The Drosophila seven-up gene, a member of the steroid receptor gene superfamily, controls photoreceptor cell fates. Cell 60, 211-224 (1990).

42. Hiromi, Y., Mlodzik, M., West, S. R., Rubin, G. M. \& Goodman, C. S. Ectopic expression of seven-up causes cell fate changes during ommatidial assembly. Development 118, 1123-1135 (1993).

43. Hoshizaki, D. K. et al. Embryonic fat-cell lineage in Drosophila melanogaster. Development 120, 2489-2499 (1994).

44. Kerber, B., Fellert, S. \& Hoch, M. Seven-up, the Drosophila homolog of the COUP-TF orphan receptors, controls cell proliferation in the insect kidney. Genes \& Dev. 12, 1781-1786 (1998).

45. Lo, P. C. \& Frasch, M. A role for the COUP-TF-related gene seven-up in the diversification of cardioblast identities in the dorsal vessel of Drosophila. Mech. Dev. 104, 49-60 (2001).

46. Sudarsan, V., Pasalodos-Sanchez, S., Wan, S., Gampel, A. \& Skaer, H. A genetic hierarchy establishes mitogenic signalling and mitotic competence in the renal tubules of Drosophila. Development 129, 935-944 (2002).

47. Tan, A. \& Palli, S. R. Identification and characterization of nuclear receptors from the red flour beetle, Tribolium castaneum. Insect Biochem. Mol. Biol. 38, 430-9 (2008).

48. Xu, J., Tan, A. \& Palli, S. R. The function of nuclear receptors in regulation of female reproduction and embryogenesis in the red flour beetle, Tribolium castaneum. J. Insect Physiol. 56, 1471-80 (2010).

49. Zelhof, A. C., Yao, T. P., Chen, J. D., Evans, R. M. \& McKeown, M. Seven-up inhibits ultraspiracle-based signaling pathways in vitro and in vivo. Mol. Cell. Biol. 15, 6736-6745 (1995).

50. Miura, K., Zhu, J., Dittmer, N. T., Chen, L. \& Raikhel, A. S. A COUP-TF/Svp homolog is highly expressed during vitellogenesis in the mosquito Aedes aegypti. J. Mol. Endocrinol. 29, 223-238 (2002).

51. Zhu, J., Miura, K., Chen, L. \& Raikhel, A. S. Cyclicity of mosquito vitellogenic ecdysteroid-mediated signaling is modulated by alternative dimerization of the RXR homologue Ultraspiracle. Proc. Natl. Acad. Sci. USA 100, 544-549 (2003).

52. Zhu, J., Chen, L. \& Raikhel, A. S. Posttranscriptional control of the competence factor betaFTZ-F1 by juvenile hormone in the mosquito Aedes aegypti. Proc. Natl. Acad. Sci. USA 100, 13338-13343 (2003).

53. Glass, C. K. \& Rosenfeld, M. G. The coregulator exchange in transcriptional functions of nuclear receptors. Genes Dev. 14, 121-41 (2000).

54. Mané-Padrós, D. et al. The hormonal pathway controlling cell death during metamorphosis in a hemimetabolous insect. Dev. Biol. 346, 150-160 (2010).

55. Broadus, J., McCabe, J. R., Endrizzi, B., Thummel, C. S. \& Woodard, C. T. The Drosophila beta FTZ-F1 orphan nuclear receptor provides competence for stage-specific responses to the steroid hormone ecdysone. Mol. Cell 3, 143-149 (1999).

56. Li, C. et al. Conserved molecular mechanism for the stage specificity of the mosquito vitellogenic response to ecdysone. Dev. Biol. 224, 96-110 (2000)

57. Maestro, O., Cruz, J., Pascual, N., Martín, D. \& Bellés, X. Differential expression of two RXR/ultraspiracle isoforms during the life cycle of the hemimetabolous insect Blattella germanica (Dictyoptera, Blattellidae). Mol. Cell. Endocrinol. 238, 27-37 (2005).

58. Martín, D., Piulachs, M. D. \& Raikhel, A. S. A novel GATA factor transcriptionally represses yolk protein precursor genes in the mosquito Aedes aegypti via interaction with the CtBP corepressor. Mol. Cell. Biol. 21, 164-74 (2001).

\section{Acknowledgements}

Support for this research was provided by the Spanish MINECO (grants BFU2009-10571 and CGL2014-55786-P to D.M. and CGL2012-36251 and CGL2015-64727-P to X.B.) and by the Catalan Government (2014 SGR 619 to D.M., J.L.M. and X.B.). The research has also benefited from FEDER funds. F.B.-C. is a recipient of a predoctoral research Grant from the MICINN. C.N. is a recipient of a postdoctoral research grant from CSIC. O.M. is recipient of a pre-doctoral research grant from Generalitat de Catalunya.

\section{Author Contributions}

Conception and design of the project was done by F.B.-C., X.B. and D.M. F.B.-C., C.N., J.L.M. and O.M. performed experiments. The analysis of the data was conducted by F.B.-C., X.B. and D.M. D.M. wrote the manuscript. All authors approved the final manuscript. 


\section{Additional Information}

Supplementary information accompanies this paper at http://www.nature.com/srep

Competing financial interests: The authors declare no competing financial interests.

How to cite this article: Borras-Castells, F. et al. Juvenile hormone biosynthesis in adult Blattella germanica requires nuclear receptors Seven-up and FTZ-F1. Sci. Rep. 7, 40234; doi: 10.1038/srep40234 (2017).

Publisher's note: Springer Nature remains neutral with regard to jurisdictional claims in published maps and institutional affiliations.

(c) (i) This work is licensed under a Creative Commons Attribution 4.0 International License. The images or other third party material in this article are included in the article's Creative Commons license, unless indicated otherwise in the credit line; if the material is not included under the Creative Commons license, users will need to obtain permission from the license holder to reproduce the material. To view a copy of this license, visit http://creativecommons.org/licenses/by/4.0/

(C) The Author(s) 2017 